\title{
Calidad de Vida del Personal de Salud y su Relación con el Ausentismo
}

\author{
QUALITY OF LIFE OF HEALTH PERSONNEL AND THEIR RELATIONSHIP TO ABSENTEEISM
}

\section{Carolina Vidal Gamboa', Ingrid Palavecino Sáez ${ }^{1}$, Patricia Moya Rivera ${ }^{1}$, Carol Toro Huerta ${ }^{1}$, Lorena Hoffmeister Arce ${ }^{1}$}

1. Escuela de Salud Pública Universidad Mayor. Santiago, Chile.

\section{RESUMEN}

Objetivo: Evaluar la relación entre la calidad de vida del personal de salud y el ausentismo en un hospital de Chile. Métodos: Estudio de corte transversal. La población de estudio corresponde a funcionarios del Hospital de Nueva Imperial. Se aplicó el instrumento de Calidad de Vida Profesional (CVP-35) y se recolectaron características demográficas, condiciones de empleo e índices de ausentismo por licencias médicas. Se realizó un modelo logístico para identificar qué factores se asocian al ausentismo. Un modelo de binomial negativo cero inflado para evaluar factores asociados con la frecuencia de licencias médicas y al número de días generados. Los modelos se ajustaron por sexo y edad, y las dimensiones del CVP35. Resultados: El porcentaje de respuesta del CVP-35 fue de $63,6 \%$. Un $67,4 \%$ corresponde a mujeres con edad media de 39,1 años. De los 352 trabajadores, 149 presentaron ausentismo por licencia médica. La mediana en los días de ausentismo es de 15 días. Presentar bastante o mucha carga de trabajo aumenta la chance de ausentarse, OR 1,29 IC95\% (1,01-1,64). La edad es un factor que se encuentra asociado a los tres índices de ausentismo evaluados, aumentando sus resultados. Discusión y conclusiones: Existe una relación entre la calidad de vida del personal de salud y el ausentismo. El ausentismo laboral es un claro síntoma de problemas en la gestión de los recursos humanos dentro de una organización.

(Vidal C, Palavecino I, Moya P, Toro C, Hoffmeister L, 2017. Calidad de Vida del Personal de Salud y su Relación con el Ausentismo. Cienc Trab. Sep-Dic; 19 [60]: 188-193).

Palabras claves: CALIDAD DE VIDA LABORAL, AUSENTISMO, PERSONAL DE SALUD, HOSPITAL.

\section{ABSTRACT}

Objective: To evaluate the relationship between the quality of life of health personnel and absenteeism in a hospital in Chile. Methods: Cross-sectional study. The population corresponds to health personnel of the Hospital of Nueva Imperial. The instrument of Quality of Professional Life (CVP-35) was applied and demographic characteristics, employment conditions and absenteeism indexes for medical licenses were collected. A logistic model was developed to identify which factors are associated with absenteeism. A zero-inflated negative binomial model to evaluate factors associated with the frequency of medical licenses and the number of days generated. The models were adjusted by sex and age, and the dimensions of the CVP-35. Results: The response rate of CVP-35 was 63.6\%. 67.4\% were women with a mean age of 39.1 years. Of the 352 workers, 149 were absent from medical leave. The median on absentee days is 15 days. Presenting a lot or a lot of workload increases the chance of being absent, OR 1.29 95\% CI (1.01-1.64)". Age is a factor that is associated with the three absenteeism indices evaluated, increasing their results. Discussion and Conclusions: There is a relationship between the quality of life of health personnel and absenteeism. Absenteeism at work is a clear symptom of problems in the management of human resources within an organization.

Key words: QUALITY OF WORKING LIFE, ABSENTEEISM, HEALTH PERSONNEL, HOSPITAL.

\section{INTRODUCCIÓN}

El ausentismo laboral es un fenómeno de preocupación constante para las organizaciones tanto públicas como privadas, en países desarrollados y en vías de desarrollo. Según el informe Human Resources Management Country Profiles, que dio a conocer la

\author{
Correspondencia / Correspondence: \\ Carol Toro Huerta \\ José Toribio Medina \#38 Santiago, Chile \\ e-mail: salud.publica@umayor.cl \\ Tel:+562 223281749
}

Recibido: 15 de Mayo de 2017 / Aceptado: 12 de Septiembre de 2017
Organización para la Cooperación y el Desarrollo Económico (OCDE) en diciembre de 2012, Chile ocuparía el primer lugar con el mayor ausentismo laboral por enfermedad en funcionarios públicos del país, alcanzando un promedio anual de 15,7 días por trabajador durante el 2010, siendo el más alto para los estándares de la OCDE que promedian 11 días por trabajador al año. Además, Chile posee el promedio anual de horas trabajadas más alto de la OCDE, con un promedio 2.048 horas de trabajo al año, muy por encima del promedio de la OCDE de 1.745 horas. ${ }^{1}$ En Chile, el ausentismo laboral por causa médica es la primera de las tres causas que generan mayor gasto público en salud. ${ }^{2}$

Los problemas que las personas enfrentan en su vida laboral son factores de riesgo importantes para su desarrollo físico y mental. ${ }^{3}$ Las causas que originan la ausencia de un trabajador de su puesto de trabajo son múltiples, pero la más frecuente es la 
ausencia por enfermedad, que junto a los accidentes laborales y particulares logran constituir hasta tres cuartas partes del ausentismo en todos los sectores de actividad. ${ }^{4}$

En particular, el entorno laboral del personal de salud se caracteriza por estar en cambio constante y generando demanda y estrés excesivo, con lo cual la institución se ve afectada negativamente. Mesa y Kaempffer ${ }^{5}$, compararon en Chile el ausentismo entre trabajadores hospitalarios, de minas, de la industria y de las universidades, y hallaron la tasa de incapacidad más alta entre los trabajadores hospitalarios. ${ }^{5}$ El ausentismo es un factor que causa problemas a los profesionales de salud, dado que involucra la ausencia de un profesional en el equipo, sobrecargando el trabajo de los demás, exigiendo un ritmo más acelerado y responsabilizándolo por un volumen mayor de trabajo. Esa sobrecarga podría perjudicar la salud del trabajador, ocasionando desgaste físico, psicológico y social. ${ }^{6}$ La evidencia ha identificado que las características propias del trabajo por turnos, tales como la duración del turno, el número total de horas semanales, el trabajo nocturno y las oportunidades de descanso pueden tener un impacto en el desempeño laboral y en el bienestar de los empleados. ${ }^{7}$

El género y la edad son variables que influyen en la solicitud y duración de las licencias médicas curativas. Las mujeres siempre se ausentan más que los hombres, sin importar su cobertura. Es interesante que el riesgo aumente con la edad, por lo que podría inferirse que realmente las licencias están asociadas a indicaciones médicas pertinentes debido a que, a mayor edad, hay mayor prevalencia de enfermedades. ${ }^{8,9}$

El objetivo del siguiente estudio fue evaluar la relación entre la calidad de vida del personal de salud y el ausentismo laboral en un hospital de Chile.

\section{MÉTODOS}

\section{Diseño y población de estudio:}

El diseño de estudio es de corte transversal. La población corresponde a funcionarios titulares, a contrata y honorarios del Hospital Intercultural de Nueva Imperial, del Servicio de Salud Araucanía Sur. El tamaño de la población corresponde a 352 funcionarios, los cuales se estratificaron en 4 grupos. Grupo I incluyó a los profesionales médicos, odontólogos y químicos ( $\mathrm{n}=55)$; Grupo II, enfermeras, matronas, nutricionistas, psicólogos, kinesiólogos y asistentes sociales ( $\mathrm{n}=91$ ); Grupo III, compuesto por técnicos en enfermería y auxiliar paramédicos $(\mathrm{n}=132)$. Finalmente el Grupo IV conformado por auxiliares de servicio y conductores $(n=74)$.

\section{Variables de estudio y trabajo de campo}

El instrumento utilizado fue la encuesta autoadministrada de Calidad de Vida Laboral CVP-35. Consta de 35 preguntas cerradas que miden tres dimensiones: (a) Motivación intrínseca, la cual valora la motivación intrínseca del trabajador por su trabajo; (b) Carga laboral que valora la percepción del trabajador frente a las demandas de la institución y (c) Apoyo directivo que consiste en evaluar el apoyo emocional percibido por parte de los directivos hacia el trabajador. Cada una de las preguntas se responde en una escala del 1 al 10, a las que se superponen las categorías "Nada" (valores 1 y 2), categoría "Algo" (valores 3, 4 y 5), "Bastante" (valores 6, 7 y 8) y "Mucho" (valores 9 y 10). ${ }^{10}$ Este cuestionario está validado en el contexto hospitalario chileno. ${ }^{11}$
El cuestionario fue entregado a todos los funcionarios del Hospital Nueva Imperial entre los meses de noviembre y diciembre de 2015. Se utilizó un sistema de devolución que garantizó la confidencialidad de las respuestas. Junto al cuestionario, se entregó un instrumento con preguntas de respuesta directa en el cual se recolectó información relacionada con características demográficas como edad, sexo y condiciones de empleo.

Para el registro de ausentismo laboral por licencias médicas de los funcionarios, se revisaron registros informáticos del hospital dos veces por semana durante un periodo de seis meses (enerojunio de 2016). Este registro informático de licencias médicas entrega información de duración de cada licencia médica, fecha de inicio y término. Con ello se creó una base de datos que permitió realizar un seguimiento de los funcionarios y calcular tres índices de ausentismo, prevalencia (se ausentó o no durante el periodo de estudio), frecuencia (número de licencias médicas presentadas durante el estudio) y gravedad (número de días generados por la licencia médica).

\section{Plan de análisis}

Se realizó una descripción de los participantes del estudio mediante tablas de frecuencias. Se estimó la media y desviación estándar para cada ítem y dimensión del instrumento CVP-35. Se estimó la proporción de trabajadores que presentó licencias médicas y se graficó la distribución de días perdidos por licencias médicas. Para evaluar la relación entre calidad de vida profesional y ausentismo laboral se realizaron tres modelos lineales generalizados, asociados a la prevalencia, frecuencia y gravedad. Se utilizó un modelo de regresión logística para identificar los factores del CVP-35 asociados a la probabilidad de presentar licencia médica ajustado por sexo y edad. A través del modelo de regresión binomial negativo cero inflado se evaluaron los factores asociados a la frecuencia de licencias médicas y al número de días de licencias médicas (gravedad de la licencia). Para valorar la utilización de este modelo se realizó el test de Vuong, el cual contrasta la utilización de un modelo binomial negativo-cero inflado con un binomial negativo estándar. El ajuste de modelos cuenta con estimaciones robustas de la varianza como lo recomiendan Cameron \& Trivedi. ${ }^{12}$ Los análisis se realizaron en el software STATA 13.0.

\section{Aspectos éticos}

El presente estudio fue evaluado y aprobado por el Comité de Ética de la Dirección de Servicio de Salud Araucanía Sur.

\section{RESULTADOS}

Se recibieron 224 encuestas de las 339 distribuidas; no fue posible entregar el cuestionario a trece personas, el porcentaje de respuesta fue de 63,6\%. Según actividad, el grupo IV y grupo III presentaron la más alta participación en el estudio con un 93,2\% y 68,9\%, respectivamente, mientras el grupo I presentó la participación más baja. Las características de la población de estudio se observan en la Tabla 1. Un 67,4\% corresponde a mujeres con una edad media de 39,1 , similar a los hombres que presentaron una media de 40,3 años. Casi un 40\% presenta una antigüedad laboral inferior a los 6 años.

En la Tabla 2 se muestran la descripción de cada item y dimen- 
Tabla 1.

Descripción de la Población de estudio.

$\begin{array}{lccc} & & \text { Frecuencia } & \text { Porcentaje } \\ \text { Sexo } & \text { Hombre } & 73 & (\%) \\ & \text { Mujeres } & 151 & 32,6 \\ \text { Grupo de edad } & \text { Total } & 224 & 67,4 \\ & 21-30 & 63 & 100 \\ & 31-50 & 116 & 28,1 \\ & 51-60 & 34 & 51,8 \\ \text { Actividad que realiza } & \text { o más años } & 11 & 15,2 \\ & \text { Total } & 224 & 4,9 \\ & \text { Grupo 1 } & 9 & 100 \\ & \text { Grupo 2 } & 55 & 4 \\ \text { Tipo de contrato } & \text { Grupo 3 } & 91 & 24,6 \\ & \text { Grupo 4 } & 69 & 40,6 \\ & \text { Total } & 224 & 30,8 \\ & \text { Contrata } & 124 & 100 \\ & \text { Titular } & 88 & 55,4 \\ \text { Antigüedad laboral } & \text { Honorarios } & 12 & 39,3 \\ & \text { Total } & 224 & 5,4 \\ & \text { 0-5 años } & 88 & 100 \\ & \text { 6-10 años } & 51 & 39,3 \\ & \text { 11-20 años } & 49 & 22,8 \\ & \text { 21-30 años } & 20 & 21,9 \\ & \text { más de 30 años } & 16 & 8,9 \\ & \text { Total } & 224 & 7,1 \\ & & & 100\end{array}$

Figura 1.

Distribución de las dimensiones del CVP-35.

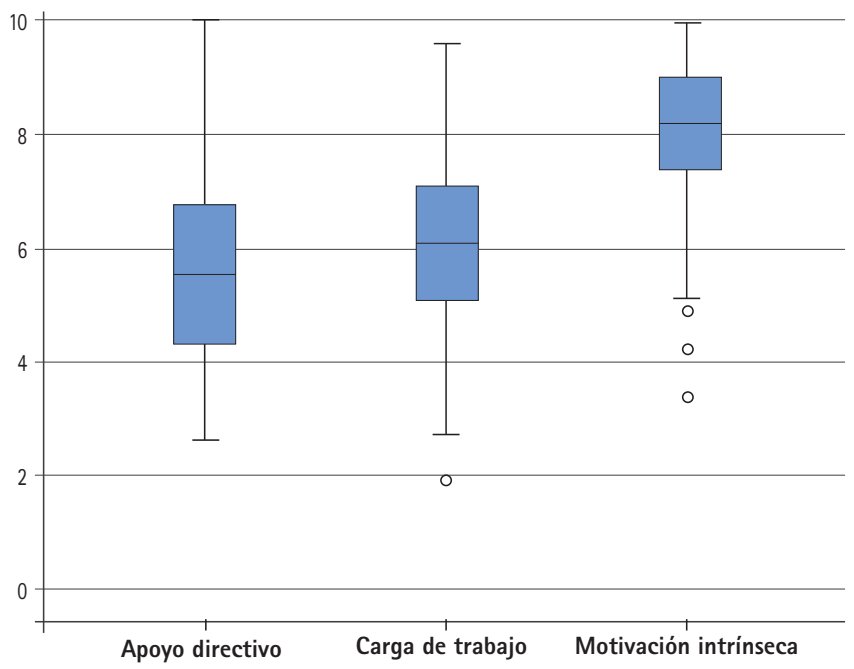

sión del cuestionario CVP-35. El factor de apoyo directivo presenta una puntuación media de 5,72 IC95\% (5,49-5,96), los ítems con un valor medio-menor corresponden a la posibilidad de promoción con 4,19 IC95\% (3,89-4,48) y satisfacción con el sueldo con valor medio de 4,65 IC95\% (4,39-4,92). El factor carga de trabajo obtiene una media de 6,20 IC95\% (5,98-6,41); las preguntas que obtienen un valor medio más alto corresponden a la cantidad de trabajo y carga de responsabilidad, con una puntuación media de 8,40 IC95\% $(8,20-8,60)$ y 7,15 IC95\% $(6,87-7,47)$, respectivamente. Finalmente, el último factor que corresponde a motivación intrínseca obtuvo un puntaje de 8,12 IC95\% (7,96-8,29). La Figura 1 muestra el gráfico de caja para cada una de estas dimensiones.
Tabla 2.

Descripción de la puntuación media de las 35 preguntas del cuestionario de Calidad de vida profesional CVP-35.

\begin{tabular}{|c|c|c|}
\hline Factores e items & $\mathrm{N}$ & Media e IC (95\%) \\
\hline Factor de Apoyo Directivo & 195 & $5,72(5,49-5,96)$ \\
\hline Posibilidad de promoción. & 209 & $4,19(3,89-4,48)$ \\
\hline $\begin{array}{l}\text { Mi empresa trata de mejorar la calidad de vida } \\
\text { de mi puesto de trabajo. }\end{array}$ & 223 & $4,65(4,39-4,92)$ \\
\hline Satisfacción con el sueldo. & 224 & $4,65(4,39-4,92)$ \\
\hline Reconocimiento de mi esfuerzo. & 221 & $5,07(4,74-5,40)$ \\
\hline Recibo información de los resultados de mi trabajo. & 224 & $5,61(5,28-5,95)$ \\
\hline Es posible que mis propuestas sean escuchadas. & 223 & $5,35(5,04-5,66)$ \\
\hline Posibilidad de ser creativo. & 222 & $6,11(5,80-6,43)$ \\
\hline Apoyo de mis jefes. & 221 & $6,67(6,32-7,01)$ \\
\hline o libertad de $d$ & 224 & $5,40(5,07-5,74)$ \\
\hline Posibilidad de expresar lo & 223 & $6,17(5,86-6,47)$ \\
\hline Variedad en mi trabajo. & 222 & $6,05(5,74-6,35)$ \\
\hline Apoyo de mis compañeros. & 221 & $7,17(6,88-7,47)$ \\
\hline Factor de Motivaciór & 213 & $8,12($ \\
\hline mi equipo. & 221 & $7,20(6,90-7,51)$ \\
\hline el tipo de trabajo. & 222 & $7,74(7,49-7,99)$ \\
\hline Motiva & 223 & $7,62(7,33-7,90)$ \\
\hline Ganas & 223 & $7,74(7,46-8,02)$ \\
\hline gulloso de & 224 & $8,67(8,45-8,90)$ \\
\hline cer mi trabajo act & 222 & 28-6,91) \\
\hline a la vida de otras personas. & 221 & $, 06-9,38)$ \\
\hline Apoyo d & 221 & $9,26(9,07-9,45)$ \\
\hline citado para & 221 & $8,80(8,60-8,99)$ \\
\hline Carga de Trabajo & 201 & $6,20(5,98-6,41)$ \\
\hline tengo. & 222 & $8,40(8,20-8,60)$ \\
\hline Prisas y agobios por falta de tiempo. & 216 & $7,15(6,84-7,47)$ \\
\hline Carga de responsabilidad. & 220 & $8,01(7,75-8,27)$ \\
\hline Estrés ( & 217 & ;59-7,28) \\
\hline alizar la cantidad de traba & 219 & $6,51(6,19-6,84)$ \\
\hline Presión recibida para mantener la calidad de mi trabajo. & 220 & $6,77(6,45-7,10)$ \\
\hline Interrupciones molestas. & 221 & $5,35(4,99-5,70)$ \\
\hline ivas para mi salud. & 221 & $5,20(4,82-5,59)$ \\
\hline hi vida personal. & 219 & $5,02(4,65-5,38)$ \\
\hline Incomodidad fisica en el trabajo. & 220 & $4,55(4,19-4,90)$ \\
\hline Conflictos con otras personas de mi trabajo. & 219 & $3,01(2,70-3,33)$ \\
\hline Me desconecto al acabar la jornada laboral. & 219 & $6,52(6,14-6,89)$ \\
\hline Calidad de vida profesional. & 215 & $6,31(6,01-6,60)$ \\
\hline $\begin{array}{l}\text { raerse del tra } \\
0 .\end{array}$ & 220 & $6,52(6,18-6,86)$ \\
\hline
\end{tabular}

De los 352 trabajadores, 149 (42,3\%) presentaron ausentismo por licencia médica. La distribución de frecuencia de licencia junto con los días de ausentismo de los trabajadores que presentaron ausentismo se observa en la Figura 2. La frecuencia de licencias médicas presenta una mediana de 1 licencia con un rango que va de 1 licencia como mínimo a 8 como máximo, mientras que valor de la mediana en los días de ausentismo es de15 días, con un rango de 4 a 155 días.

El análisis multivariado asociado a la probabilidad de presentar ausentismo por licencia médica se observa en la Tabla 3, mostrando que las mujeres aumentan la probabilidad de ausentarse respecto a los hombres OR: 2,02 IC95\%:(1,08-5,14). Otro factor corresponde a la carga de trabajo: la mayor carga de trabajo aumenta la chance de ausentarse, OR 1,29 IC95\% (10,1-1,64). Respecto a la frecuencia de licencias médicas, se observa que los trabajadores de mayor edad presentan una asociación significativa, vale decir que los trabajadores de 50 años o más aumentan en un 85\% (p-valor: 
Figura 2.

Distribución de la frecuencia de licencias médicas presentadas y dias totales ausentismo.
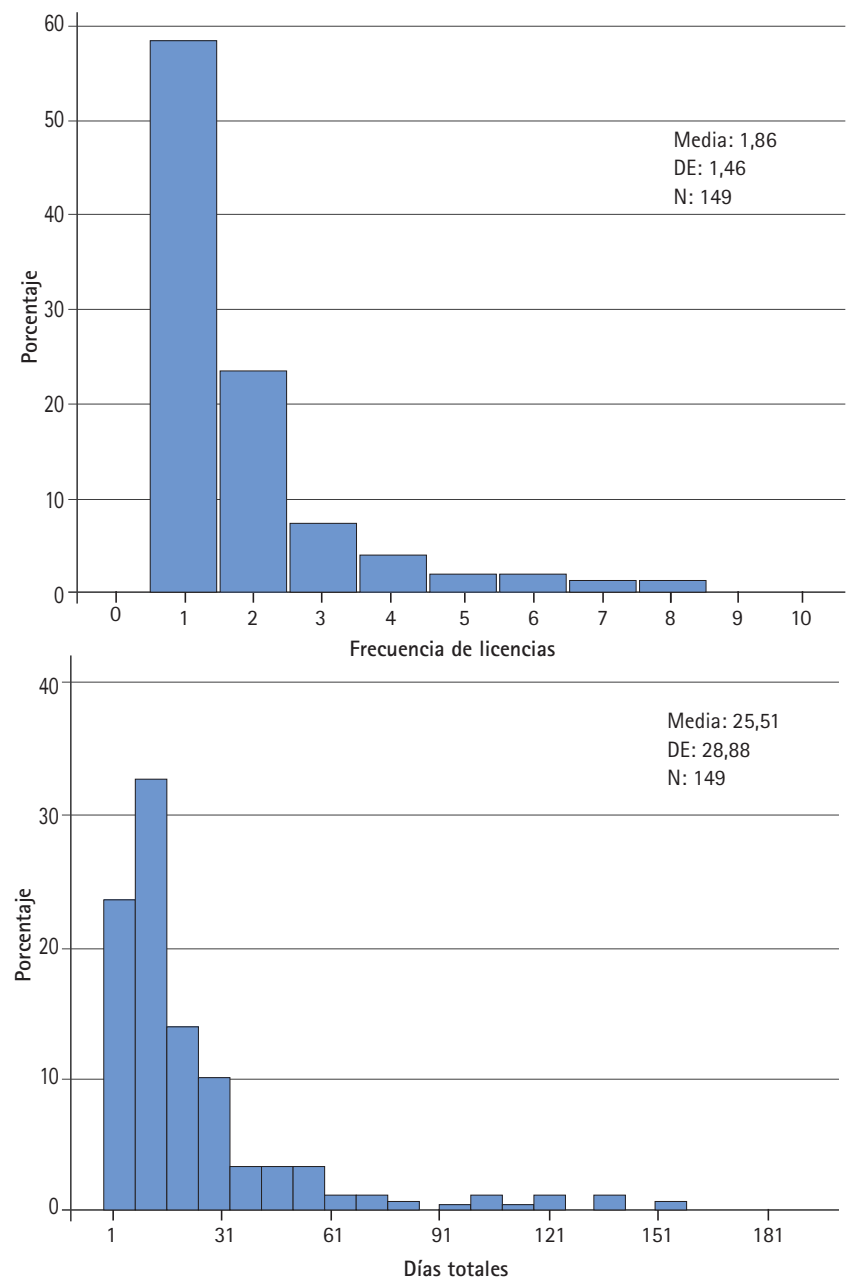

0,05) el número de licencias médicas presentadas respecto a los trabajadores menores de 30 años. El apoyo directivo se asoció a una disminución en los días totales asociados a las licencias médicas IRR: 0,87 IC95\% (0,75-0,99).

\section{DISCUSIÓN}

La calidad de vida profesional es una variable de interés para el sistema sanitario y, por tanto, debe ser medida con precisión y exactitud. ${ }^{13}$ Existen varios instrumentos para medir la calidad de vida profesional y que se encuentran validadas en lengua castellana. Nuestro estudio utilizó el CVP-35, validado para contexto hospitalario en Chile. ${ }^{11}$ Respecto a los resultados de este instrumento, se observó que la dimensión que presenta un valor medio mayor corresponde a la motivación intrínseca con un puntaje de 8,12 IC95\% (7,96-8,29), valor levemente superior al observado por Arrué y cols.13 en un Hospital de la Región Metropolitana, donde obtuvo para la misma dimensión un puntaje medio de 7,8 IC95\% (7,6-7,9). Otros estudios asociados a profesionales de salud de atención primaria muestran que la motivación intrín- seca es moderadamente alta, sobre todo en las mujeres y no se relaciona con la edad, la categoría profesional o el entorno del trabajo. ${ }^{14}$

La medición del ausentismo laboral constituye hasta hoy una problemática, la literatura reporta diferentes métodos dependiendo del área de investigación. Este estudio utilizó los índices de prevalencia, frecuencia y gravedad, los que se identifican como los más utilizados para los estudios del área de la salud.

Este estudio también evidenció un ausentismo de 42,3\%, resultado inferior al estudio de Concha L y cols. ${ }^{15}$, el cual estimó que un 67,4\% de ausentismo en el año 2012 en los trabajadores pertenecientes a los establecimientos autogestionados en red; esta diferencia puede estar asociada al tiempo de observación. Respecto a los días de ausentismo, un estudio realizado en el Servicio de Salud de Nuble ${ }^{9}$ identifica una mediana de 8 dias en hombres y de 12 días en mujeres que presentaron al menos una licencia médica. Este estudio evidenció una mediana de 15 días. Concha L y cols. ${ }^{15}$ también muestran que existen diferencias para las variables de sexo, edad, calidad jurídica, remuneración cargo y turno entre los trabajadores ausentados y no ausentados, mientras que las variables de estamento y horas semanales de contratación no presentaron diferencias. El estudio de Soto y cols. también muestra que género y edad son variables que influyen para la solicitud y duración de las licencias médicas curativas. ${ }^{9}$ El presente estudio evidencia que las mujeres se asocian a un mayor ausentismo (esta asociación no se mantiene al evaluar los índices de frecuencia y gravedad). Mientras que la edad es un factor que se encuentra asociado a los tres índices evaluados, ser un trabajador de 50 años o más se asocia a una mayor probabilidad de ausentarse, a un mayor número de frecuencia y a más días de ausentismo. Respecto a las dimensiones de calidad de vida laboral se observa que la carga de trabajo se asocia con la probabilidad de ausentismo, mientras que el apoyo directivo disminuye los dias totales de ausentismo. Estudios han evidenciados que los condiciones ambientales del trabajo y de la organización son mejores predictores del ausentismo que características demográficas. ${ }^{16}$ Un estudio realizado en Chile muestra que el ausentismo está influenciado por la satisfacción laboral y la cultura organizacional. ${ }^{17}$

El ausentismo laboral es un claro sintoma de problemas en la gestión de los recursos humanos dentro de una organización. Una de las primeras tareas que tienen los Servicios de Salud del país y su red asistencial es visualizar que el principal recurso con el que cuentan para cumplir sus objetivos son sus trabajadores, los cuales, con herramientas apropiadas, y una buena calidad de vida laboral contribuirán de manera significativa a la producción de las instituciones de salud. En este sentido, velar por las buenas condiciones de trabajo es una de las principales estrategias para gestionar e intervenir el ausentismo laboral. ${ }^{15}$ La planificación sistemática de la fuerza de trabajo es un elemento clave de la gestión de la calidad de la atención sanitaria. ${ }^{18}$ Los esfuerzos para reducir el agotamiento producido por el trabajo y un aumento del apoyo psicológico en los trabajadores de la salud apoyan la motivación con que este se desarrolla, proporcionando mejores servicios. Por lo que se incrementaria la productividad personal y se obtendrá ganancia en el sentido institucional. ${ }^{3}$

La literatura reporta que la implementación de programas de soporte o entrenamiento físico multifacéticos puede producir resultados positivos en cuanto a la reducción del ausentismo de enfermería hospitalaria. ${ }^{19}$ También han ganado cada vez más atención las estrategias preventivas hacia la ausencia por enfermedad, 
Tabla 3.

Modelo lineales generalizados para identificar factores asociados a ausentismo, frecuencia de licencias y al número de días de ausentismo.

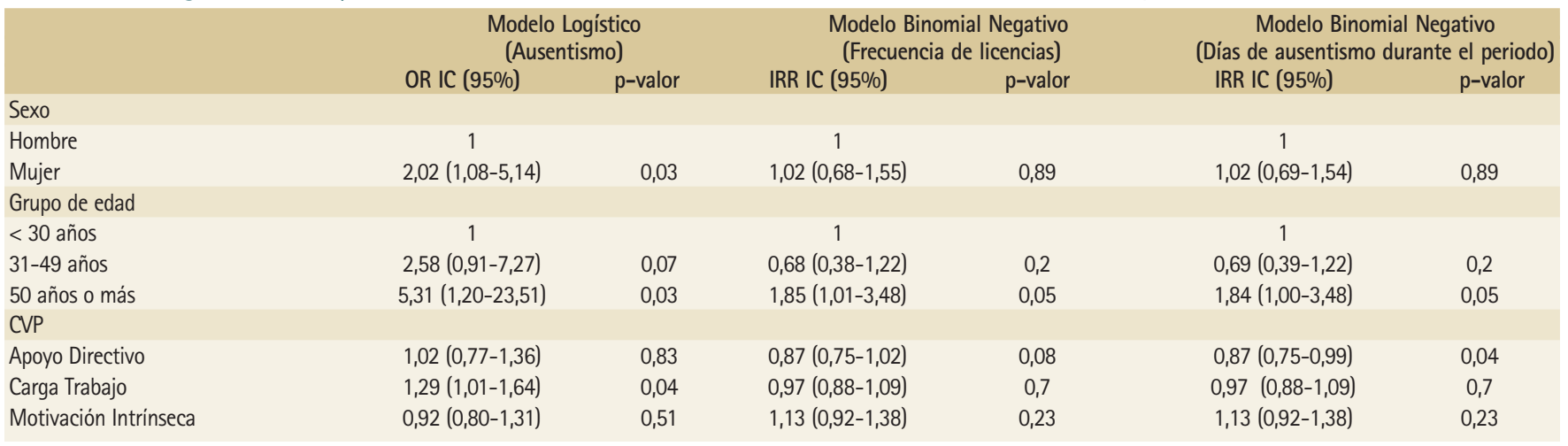

que se concentran en el rediseño de empleos para mejorar las características de varios de ellos. ${ }^{20}$ La investigación sobre la reorganización de la prestación de servicios de salud sugiere que el cambio a la atención en equipo se acompaña de estrés y preocupaciones acerca de la claridad del rol y que los resultados varían para los proveedores en organizaciones privadas y públicas. ${ }^{21}$

Las organizaciones pueden considerar el uso de iniciativas de cambio cultural para aumentar la cortesía en el trabajo; esto incluye el apoyo al liderazgo y el modelado de los comportamientos del lugar de trabajo. Los líderes deben monitorear los niveles de personal y cargas de trabajo altas para prevenir el agotamiento emocional, que predice la rotación. La contratación y la capacitación de los supervisores deben incluir no sólo la experiencia técnica, sino también las habilidades blandas necesarias para crear entornos civiles y de apoyo. Los líderes y gerentes deben usar datos de retroalimentación de los empleados (por ejemplo, encuestas organizacionales) para aprender sobre los ambientes de trabajo y abordar las áreas de preocupación de los empleados. ${ }^{22}$ Una de las limitaciones del estudio corresponde al desconocimiento de la distribución de las causas por licencia. Estudios similares han demostrado que entre un 85 a 90\% corresponden a licencias por enfermedad común, un 5\% a descanso maternal y el 10\% restante en forma decreciente corresponde a licencias por patología de embarazo, accidente de trabajo y trayecto, enfermedad profesional. ${ }^{15,23}$ Otra limitación corresponde a la no incorporación de las condiciones de empleo como variables explicativas; estas no fueron utilizadas ya que generaban problemas de sobreparametrización de los modelos estadísticos. ${ }^{24}$ Las fortalezas del estudio corresponden a la medición individual de la percepción de la calidad de vida de los trabajadores de un hospital público en Chile y sus resultados con los indicadores de ausentismos.

Tal como es recomendado por diversos autores ${ }^{15}$, es necesario continuar en la línea de las investigaciones respecto del ausentismo por incapacidad laboral en los establecimientos de salud, incorporando otras características individuales de los trabajadores, del ambiente físico y el ambiente organizacional que influyen también en el ausentismo. El sector público de salud representa una importante fuerza laboral, por lo tanto, cualquier estudio que genere información relevante sobre sus trabajadores y a su vez repercuta en acciones positivas hacia ellos, contribuye de alguna manera a la salud de la gran mayoría de los chilenos. 


\section{REFERENCIAS}

1. Organization for Economic Co-operation and Development (OECD). Human Resources Management Country Profiles: Chile [on line]. Paris: OECD Publishing; 2012 [cited Nov 2017]. Available from: http://www.oecd.org/gov/pem/OECD HRM Profile - Chile.pdf

2. Chile. Ministerio de Hacienda. Dirección de Presupuesto. Estadisticas de Recursos Humanos del Sector Público 2003-2012. Santiago de Chile: Ministerio de Hacienda; 2013.

3. Gorgulu 0, Akilli A. The determination of the levels of burnout syndrome, organizational commitment, and job satisfaction of the health workers. Niger J Clin Pract. 2017; 20(1):48-56.

4. De Antonio M. Problemática multifactorial del absentismo laboral, el presentismo y la procrastinación en las estructuras en que se desenvuelve el trabajador. Med Segur Trab. 2011; 57(223):111-120.

5. Mesa M, Kaempffer R. 30 Años de estudio sobre ausentismo laboral en Chile: Una perspectiva por tipos de empresas. Rev Méd Chile. 2004; 132(9):1100-1108.

6. Martinato $M$, Severo $D$, Marchand $E$, Siqueira H. Absenteismo na enfermagem: uma revisão integrativa. Rev Gaúcha Enferm. 2010; 31(1):160-166 .

7. Dall'Ora C, Ball J, Recio-Saucedo A, Griffiths P. Characteristics of shift work and their impact on employee performance and wellbeing: A literature review. Inter J Nurs Stud. 2016; 57:12-27.

8. Miranda G, Alvarado $S$, Kaufman J. Duración de las licencias médicas FONASA por trastornos mentales y del comportamiento. Rev Méd Chile. 2012; 140(2):207-213.

9. Soto $A$, Herrera R, Fuentes R. Efecto de la seguridad social en la duración del ausentismo laboral en el Servicio de Salud de Nuble: un análisis de supervivencia. Rev Méd Chile. 2015; 143(8):987-994.

10. Martín J, Alfonso Cortés J, Morente $\mathrm{M}$, Caboblanco $\mathrm{M}$, Garijo J, Rodriguez A. Caracteristicas métricas del Cuestionario de Calidad de Vida Profesional (CVP35). Gac Sanit. 2004; 18(2):129-136.

11. Arrué L, Monsalve M. Calidad de vida laboral de los funcionarios del Hospital Félix Bulnes Cerda; Servicio Metropolitano Occidente 2013. Santiago de Chile: Universidad Mayor; 2013.

12. Cameron AC, Trivedi PK. Microeconometrics using Stata. College Station (TX):
Stata Press; 2009.

13. Martín J, Gómez T, Martínez C, del Cura MI, Cabezas MC, Garcia S. Medición de la capacidad evaluadora del cuestionario CVP-35 para la percepción de la calidad de vida profesional. Aten Primaria. 2008; 40(7):327-334.

14. Cortés J, Martín J, Morente $M$, Caboblanco M, Garijo J, Rodríguez A. Clima laboral en atención primaria: ¿qué hay que mejorar?. Aten Primaria. 2003; 32(5):288-295.

15. Rojas L, Guillou M. Estudio descriptivo del ausentismo laboral en trabajadores del Sistema Público de Salud en Chile. Santiago: Universidad de Chile; 2014.

16. Farrell D, Stamm CL. Meta-Analysis of the Correlates of Employee Absence. Hum Relat. 1988; 41(3):211-227.

17. Mendoza R. Insatisfacción laboral como predictor del ausentismo en un hospital público. Rev Méd Chile. 2015; 148:1028-1033.

18. Curson J, Dell ME, Wilson R, Bosworth DL, Baldauf B. Who does workforce planning well? Workforce review team rapid review summary. Int J Health Care Qual Assur. 2010; 23(1):110-119.

19. Jesús J, Gutiérrez B, Jiménez MC, Escalera LF. Intervenciones eficaces para reducir el absentismo del personal de enfermeria hospitalario. Gac Sanit. 2013; 27(6):545-551.

20. Janssens H, Braeckman L, De Clercq B, Casini A, De Bacquer D, Kittel F, et al. The indirect association of job strain with long-term sickness absence through bullying: a mediation analysis using structural equation modeling. BMC Public Health. 2016; 16(1):851.

21. Hastings $S E$, Armitage GD, Mallinson $S$, Jackson $K$, Suter E. Exploring the relationship between governance mechanisms in healthcare and health workforce outcomes: a systematic review. BMC Health Serv Res. 2014; 14:479.

22. Yanchus NJ, Periard D, Osatuke K. Further examination of predictors of turnover intention among mental health professionals. J Psychiatr Ment HIt. 2017; 24(1):41-56.

23. Parada M, Barrios R, Rincón $E$, Mejía Z, Mora D, Padilla F. Ausentismo Laboral de Causa Médica. MedULA. 2006; 15:22-25.

24. Acosta $E$, Fernández F. Model selection via genetic algorithms illustrated with cross-country growth data. Empir Econ. 2007; 33(2):313-337. 